\title{
Design and Construction of Monocopter and its Nonlinear Control Using Photo Diode Array
}

\author{
Ahmad Safaee ${ }^{1}$, Seyed Zeinolabedin Moussavi ${ }^{2}$, \\ Mohammad Bagher Menhaj ${ }^{3}$ \\ ${ }^{1}$ Doctoral Program in Electrical and Computer Engineering, Faculty of Engineering, University \\ of Porto, Porto, Portugal (safaee.ah63@gmail.com); ${ }^{2}$ Faculty of Electrical \& Computer \\ Engineering, Shahid Rajaee Teacher Training University, Tehran, Iran (smoussavi@srttu.edu); \\ ${ }^{3}$ Faculty of Electrical Engineering, Amirkabir University of Technology, Tehran, Iran \\ (Menhaj@aut.ac.ir)
}

\begin{abstract}
Monocopter is a single-wing rotary flight system which has the capability of hovering. This flyer includes two dynamic parts in which more efficiency can be expected rather than other Micro UAVs due to the extended area of wing compared to its frame. Low capital cost and simple mechanism rather than other systems such as helicopter are the most important specifications of this flight system.

In this paper, a model of monocopter flight system has been designed and implemented. Then, a new method of monocopter control and the related hardware has been designed and constructed using photo diode array in order to define position angle of flight system. Designers and constructors have to build a digital compass with the related advantages and disadvantages for this purpose. In the next stage, the optimized flight sample was redesigned and reconstructed and the dynamic parameters of flying were set due to existed hardware restrictions. Then, different experimental tests have been carried out after hardware installation of the control system. So, the controllable flying has been implemented using this control system.
\end{abstract}

Author Keywords. Monocopter, Gyroscopic Motion, Flap, Heading Angle, Hovering, Cyclic, Photo Diode

Type: Research Article

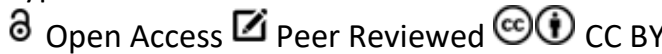

\section{Introduction}

Monocopter is a kind of rotary flight system or Gyropter which uses a single wing for flight. The main idea for building this flyer has been brought from the maple seed. When this seed asides from the maple tree, it flies precisely and regularly like a small helicopter till it comes down to the ground. In this state, the heavier part which contains the seed has the role of rotary axes and center of gravity while the terminative part operates like a wing or a propeller in which a small lift force can be produced by this rotating system and it lands slowly like a glider.

First time, the idea of building Micro UAV using this flight model was proposed by DARPA institution in 2006. Till now, few research institutes conduct their research directions to this subject. A few numbers of them can successfully build the monocopter control and supervisory system. Some of these projects with favorable outcomes have been carried out in the universities of MIT and Maryland, and the Lockheed Martin Company. 


\section{Materials and Methods}

\subsection{Flying platform}

In our project, a flight system has been designed and constructed using balsa wood for flight testing purposes. The construction of this sample has been performed after the extensive investigations in aerodynamics of gyroptors and several experimental tests in different models of monocopters (more than four models) and their operation and flight method assessments. This flyer consists of one main wing, a flap and its servomotor, a fly-bar, a brushless motor, motor speed control system, battery, flight computer system and motor strut (Figure 1). The motor strut in this system causes the main weight to be farther than the flyer rotation center and increases the rotational moment of inertia of the system, thereby providing stability to fly. Because if the moment of inertia of the rotating system increases, more gyroscopic force can be created and this force resisting the sliding motion will cause the rotation of the system be stable.

A brushless DC electrical motor with the maximum of $800 \mathrm{~g}$ thrust force has been employed as a forward driving force in this flight system. In this system, an operator has been used which is installed on the sole existed flap of the system. For this purpose, a high speed electrical servomotor has been applied. This servomotor (HSG-5084MG) is the product of Hitec Company and specifically is used for control of unmanned helicopter tail in which the commands are received with the frequency of $250 \mathrm{~Hz}$ and the response time of the system for 60 degrees of rotation is 70 milliseconds. Therefore, due to this fact that the angle of required operator in this system is about 20 degrees, it can be expected that this rate of changes is applied in the time duration of about 30 milliseconds. This time range is one of the fundamental restrictions in the control of rotating systems. The dimensional characteristics of this flyer are illustrated in Table 1.

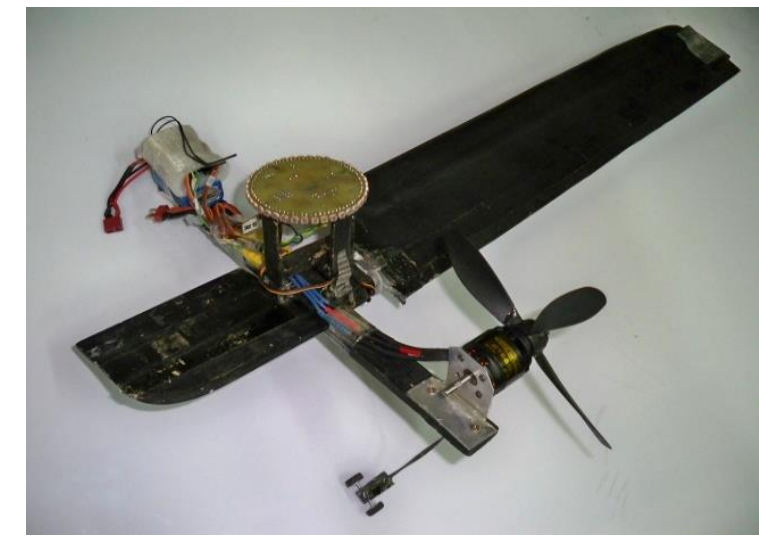

Figure 1 : Monocopter system constructed

\begin{tabular}{l|l}
\hline Frame Length & 73 Centimeters \\
Wing span & 53.5 Centimeters \\
Wing width & 10 Centimeters \\
Fly-bar length & 13 Centimeters \\
Fly-bar width & 10 Centimeters \\
Flap width & 3 Centimeters \\
Flap length & 64 Centimeters \\
Stabilizer lenght & 35.5 Centimeters \\
Weight & 450 gr \\
\hline Table 1: Dimensional characteristics of flyer
\end{tabular}




\subsection{System hardware}

The main problem in the control of rotary system is to access a fixed and precise reference for flyer heading angle. In some flying systems like helicopters, this problem is resolved by controlling the heading angle of the frame. Therefore, the control commands of flaps or cyclic commands in the system are applied relative to the frame. But in monocopter as a gyropter system, due to the rotational motion of the whole the frame, no fixed reference exists for defining the flap commands. So, it is necessary to calculate the flyer heading angle relative to the external reference by using the appropriate sensors. In the similar projects, the digital compass is used for this purpose which is consistent of magnetometer sensors and accelerometer. In these systems, the position angle of the flyer is calculated relative to the earth magnetic north. This is considered as the coordinate reference.

But in this project, a new framework has been used in which an optical (or light) resource is used as the reference of the heading angle. This reference can be used both in fixed or motional conditions. Thus, this optical resource can be available for the pilot to shift it in order to alter the commands calculated reference. So, the commands to the system for moving left, right, forward and upward will be always applied along the direction of the pilot related to the system. By shifting the position of the system or heading angle of the pilot, the command will be fixed as the viewpoint of the pilot.

Besides, it should be noted that the main target in selecting this control system for this flyer is its simple hardware rather than the hardware in digital compass. Otherwise, the sensor fusion of digital compass system, as the first effort of designers in monocopter control, could reduce the work speed and efficiency. So in the first stage, we have decided to present this new approach in order to test the control methods of monocopter system.

For this purpose, a 40-arrays of photo diode has been installed and the sensors output data has been measured by an Xmega64 microcontroller using five 8?1-multiplexer. This processor unit calculates the direct of optical resource position related to the system by selecting the maximum value among the measured data of the sensors. In this processor, the control commands which are applied by the pilot are received in the form of PWM signals via the radio control receiver. Then, due to the direction of the applied command, a sector of circular path in which the flap commands should be applied is computed. Therefore, in an appropriate time instant, the command required for servo-flap is calculated and applied to the servomotor as a PWM signal. Figure 2 illustrates the electronic circuit of the flight computer.

So, the pilot applies the commands for moving left, right, upward and downward and commands for motor speed (Throttle) with radio control. The throttle command is applied directly from radio control to the speed control system and control the flight height manually. The other commands are received by flight computer in which appropriate control operations are performed considering these commands. The schematic of system hardware is depicted in Figure 3.

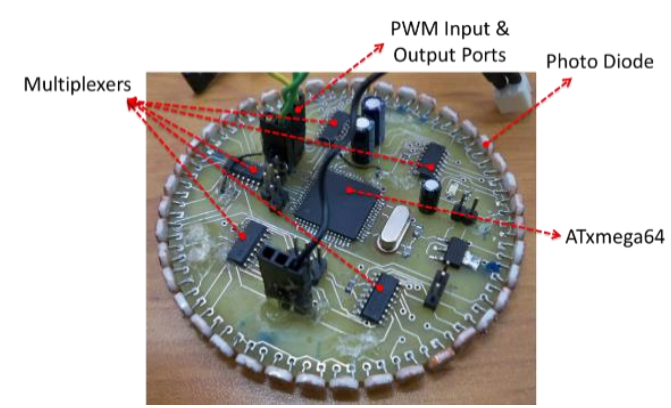

Figure 2: Flight computer system 


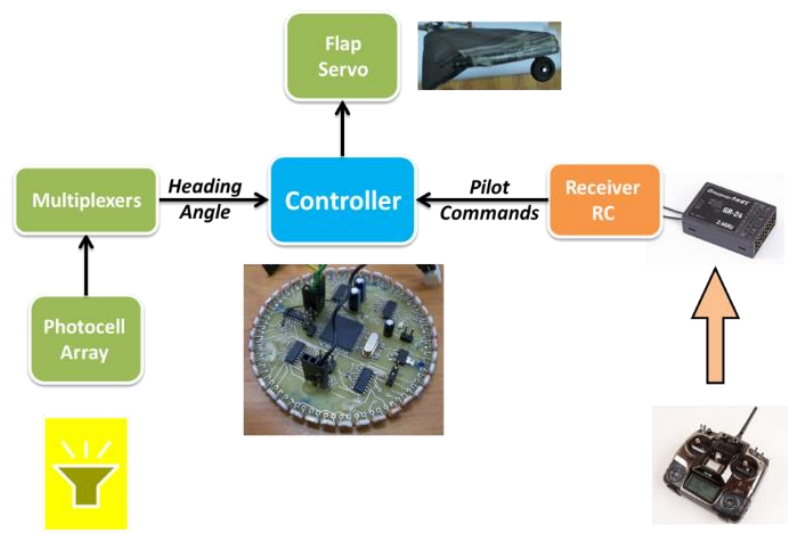

Figure 3: Equipment configuration in the system

As it was referred, the configuration of flyer different parts and equipment in the flying system influences strongly on the flying system operation. Figure 4 shows the equipment configuration in the flying system. The other important factor in providing the flight stability of these systems is the rotating speed of the flyer. Thus the more rotating speed of the flyer, more gyroscopic force will be induced to the system.

But it should be noted that if the rotating speed of the flyer becomes more, then faster and stronger operators will be required to control these systems. Then due to the restricted control hardware system, the flying system has been designed and constructed in such a way that the rotating speed in hovering is about 5 cycles per second.

The setting of central gravity in this flyer is an important and precise factor. If the central gravity of system is farther from fly bar, the cone angle of flying in the system will increase (See Figure 5) (Varshney, Chang, and Wang 2012). In this circumstance, the system stability will increase in one hand and in the other hand, the system controllability will decrease. In addition, the increase lift force of wing and fly-bar will be effective in determination of flying cone angle in the flyer (Ho and Wong 2006; Zhang et al. 2002).

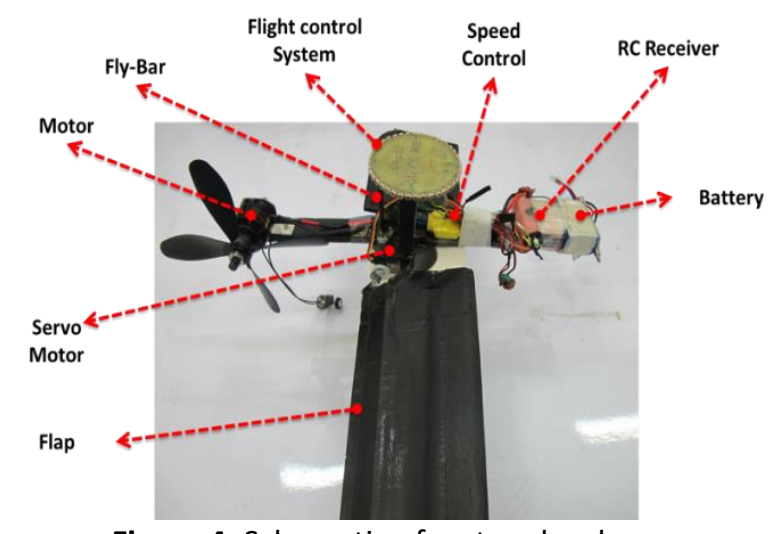

Figure 4: Schematic of system hardware

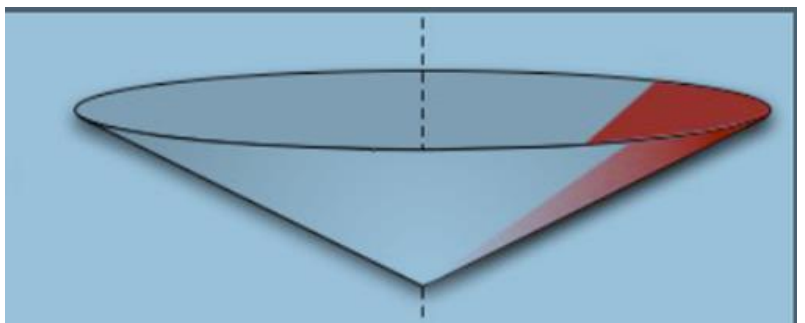

Figure 5: The flying cone angle 


\subsection{Test results}

The primary tests of this project were carried out for achieving a model with appropriate dimensions, weight and aerodynamics. After these assessments, an appropriate model for primary design of flyer has been adopted and built. In this test stage in which no control and forward system exist, the flyer was hovered from appropriate height and then the rotary of flyer in the falling situation and its weight balancing were assessed. In the second stage of the tests, the forward force was applied to the flyer in order to investigate about the quality of its take-off, the state stability and also the relationship between the motor power and the flyer weight (Figure 6). In the final stage of the tests, the optic control system and flap operator were added to the system and then some control tests were carried out on the flyer.

It should be noted that the control commands should be applied in the states of start-stop to the system control. If the control commands are applied to the system in several sequential cycles, some perturbations in the form of oscillatory motion can be found in the system. Otherwise, the system reaction speed to the control command is high so that the angle deviation in the flyer can be quickly seen after the first flap command. This angle deviation causes immediately the changes in the direction of flyer motion and consequently, changing in its position.

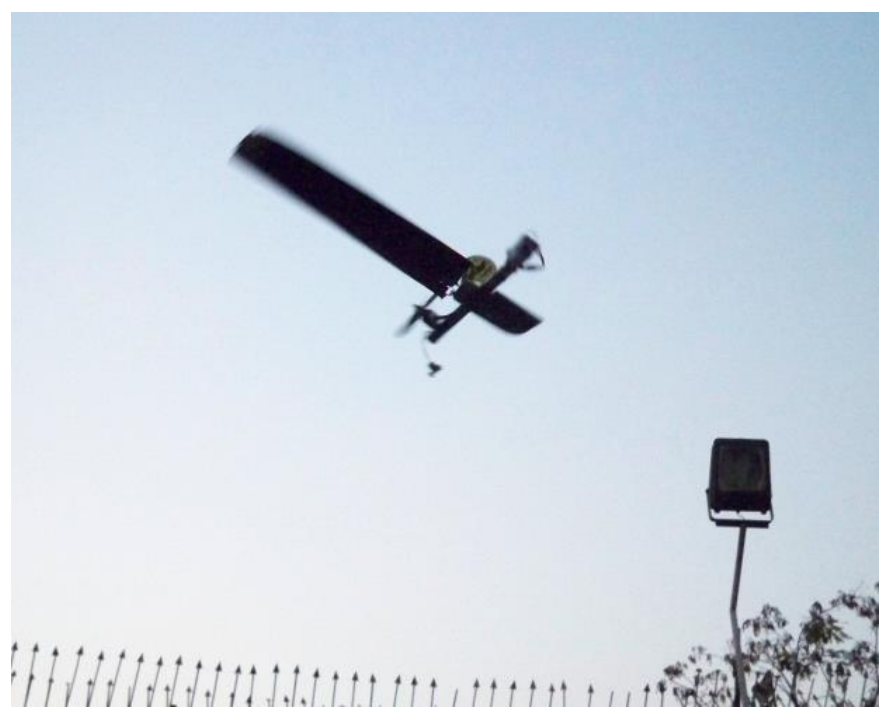

Figure 6: Monocopter system in the state of flight test without control

Figure 7 shows some photos of control tests which were performed on our monocopter system. As it is shown, the pilot is on the left side of the picture while the optic resource exists in the opposite direction (the right side of the picture).

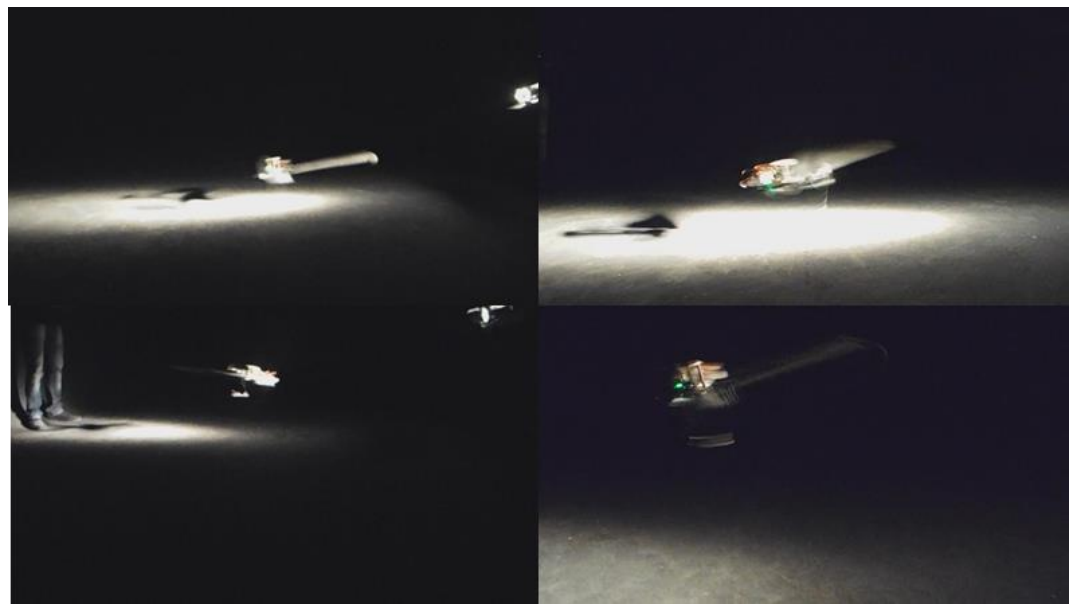

Figure 7: Monocopter system in the state of control tests 


\section{Discussion}

Considering the commands which are applied by the pilot and the heading angle of the system, the flyer flap will be activated in special times while it will not be active in other states. The main consideration in this control method is the operation speed of the servo-flap. Since due to the rotary speed of the system and considering the 60-degrees sector for flap controller command, only about 30 milliseconds time span remains for applying the command in each cycle. So, it can be stated that in this system, the rotary speed of the flyer is in the bounded (threshold) condition related to the operation speed of the servo. In the higher rotary speed, the system may completely loose its performance.

The flapping mechanism in monocopter is very similar to the helicopter cyclic system. In some systems such as helicopter, when the command is applied to one side of the flap, the system is inclined to the opposite side.

For assessing the flapping mechanism, several tests have been carried out on the stand and effectiveness of commands and system operation was fully tested (See Figure 8). It was found that the effectiveness of control commands is exactly similar to the helicopter cyclic system (Bakula et al. 2009; Hockley et al. 2010).

It was discovered in the flight test of monocopter system that due to the complex nature of gyroscopic dynamics, there is a shift phase in the system. The value of this shift phase is the function of moment of inertia and the rotary speed of flyer.

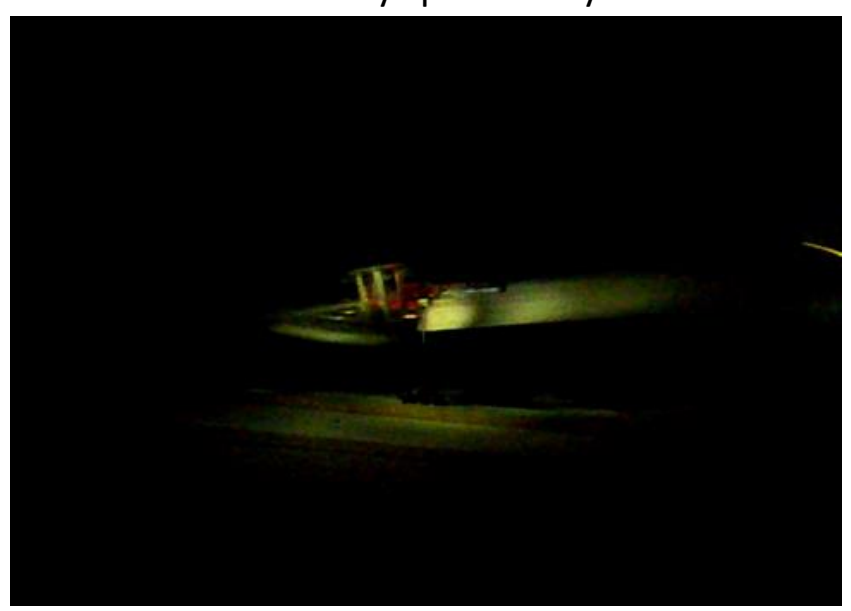

Figure 8: The performed tests on the stand

For instance in the main system, in the state in which the pilot stands on the south, in order to be ability to interpret the commands, if the commands to the forward direction is applied to the stick, the flap command will be applied when the flyer wing stands far from the pilot (i.e., the same direction that it should move towards) while the flap command is in the downward direction and the generated force due to this command is along the lift increase. This command in the helicopter system causes the deviation of flyer rotation screen to the backward. This phase shift happens also in other axes. This phenomenon indicates the 180 degrees of phase shift compared to the cyclic system of helicopter (Bakula et al. 2009; Houghton and Hoburg 2008; Kellas 2007; Obradovic et al. 2012). Figure 9 illustrates the system steering related to the control commands. The main causes for this phase shift should be explored in the rules governed on the gyroscopic phenomenon. Since in a hovering gyropter, the amount of this force is greater than the amount of the force applied from the flap command, the system reaction related to the flap command will be opposite. Of course the change in gyroscopic force due to the moment of inertia in the system or rotary speed may change this phase shift. 


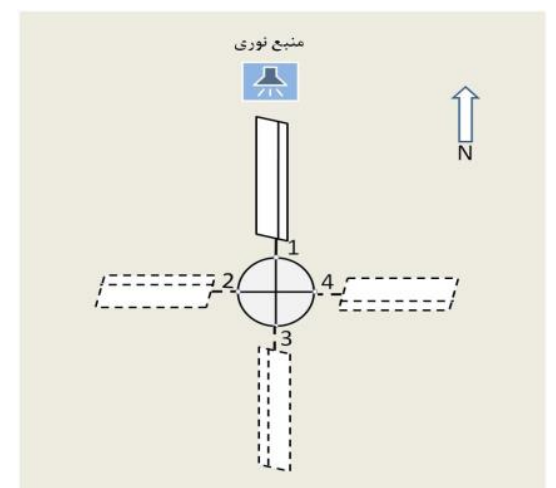

Figure 9: The schematic of phase difference of commands in the system

\section{Conclusions}

The monocopter which is built for this project has very good flight stability and to some extent is robust against the environmental perturbations such as ground effect and sided wind. Also, it was found out if the system rotary speed increases, this stability will increase. Otherwise, the rotary speed of monocopter flying is the function of system weight, wing angle of attack and the wing area. So by setting these parameters, the rotary speed of the system can be regulated due to the hardware restrictions.

The main consideration of this control method is the system requirement to the dark environment and completely isolated area from other optical resources. This problem causes the system control test to be possible only in experimental conditions. This constraint can be accounted as the main disadvantage of this control method. The first stage in developing this control system is employment of optical resource with the special spectrum and using the spectrum filters in optical receiver sensors. So, the destructive effects of environmental conditions can be reduced to high extent and the ability of controlled flight in the nonexperimental conditions can be provided.

By adding the appropriate sensors for measuring the flyer heading angle, roll and pitch angle control loops (which are flight cone angle and wing angle of attack, respectively) can be implemented. If these parameters are controlled, the flight stability can be provided without using any fly-bar. Thus, with fly-bar and motor strut omission, it can be possible to build smaller and faster samples. Since this model is naturally unstable, the implementation of angles control loops is essential for its stabilization. The sample of this flying model has been built for completing the pervious investigations and its inherent instability was observed (Jameson et al. 2007; Ulrich, Humbert, and Pines 2010).

\section{References}

Bakula, Michael, Christopher Hockley, Rajan Khatri, Christopher Kirby, Christopher Sammet, and Charles Reinholtz. 2009. "A natural evolution in flight: The design and development of the SamarEye system, A method for searching closed Quarter Environments". Paper presented at the First Symposium on Indoor Flight Issues, Mayagüez, Puerto Rico, July 21, 2009.

http://www.aerialroboticscompetition.org/assets/downloads/2009SymposiumPapers/20 09EmbryRiddle.pdf.

Ho, Derrick, and K. Wong. 2006. "Investigation of low thrust to weight ratio rotational capacity of asymmetric mono-wing configurations". Paper presented at the 28th International Congress of the Aeronautical Sciences, Brisbane, Australia, September 23-28, 2012. http://www.icas.org/ICAS_ARCHIVE/ICAS2012/PAPERS/725.PDF. 
Hockley, Christopher, Michael King, Rajan Khatri, Christopher Kirby, Christopher Sammet, Michael Bakula, and Charles Reinholtz. 2010. "Development of a monocopter for exploration of GPS-denied indoor environments". Paper presented at the Second Symposium on Indoor Flight Issues, Mayagüez, Puerto Rico, August 12, 2010. http://www.aerialroboticscompetition.org/assets/downloads/2010SymposiumPapers/ER AU_2010.pdf.

Houghton, James, and Woody Hoburg. 2008. Fly-by-wire control of a monocopter. Project Report, Experimental Projects II (course 16.622), Massachusetts Institute of Technology. https://www.researchgate.net/publication/237758639_Fly-bywire_Control_of_a_Monocopter.

Jameson, Steve, Brian Satterfield, Cortney Bolden, Ned Allen, and Hal Youngren. 2007. SAMARAI nano air vehicle - A revolution in flight. Lockheed Martin Aeronautics, Advanced Development Programs, Palmdale,

CA. https://www.researchgate.net/publication/228671803.

Kellas, Andreas. 2007. "The guided samara: design and development of a controllable singlebladed autorotating vehicle". Master's thesis, Department of Aeronautics and Astronautics, Massachusetts Institute of Technology. http://hdl.handle.net/1721.1/42047.

Obradovic, Borna, Gregory Ho, Rick Barto, Kingsley Fregene, and David Sharp. 2012. "A multiscale simulation methodology for the Samarai monocopter? UAV". Paper presented at the AIAA Modeling and Simulation Technologies Conference, Minneapolis, Minnesota, August 13-16, 2012. https://arc.aiaa.org/doi/abs/10.2514/6.2012-5012.

Ulrich, Evan R., J. Sean Humbert, and Darryll J. Pines. 2010. "Pitch and heave control of robotic Samara micro air vehicles". Journal of Aircraft 47, no. 4 (july-august): 1290-99. https://doi.org/10.2514/1.47197.

Varshney, Kapil, Song Chang, and Z. Jane Wang. 2012. "The kinematics of falling maple seeds and the initial transition to a helical motion". Nonlinearity 25, no. 1: C1. https://doi.org/10.1088/0951-7715/25/1/C1.

Zhang, Xin, Jonathan Zerihan, Andreas Ruhrmann, and Michael Deviese. 2002. "Tip vortices generated by a wing in ground effect". Paper presented at the 11th International Symposium on Applications of Laser Techniques to Fluid Mechanics, Lisbon, Portugal, July, 8-11, 2002. http://Itces.dem.ist.utl.pt/Ixlaser/Ixlaser2002/papers/paper_4_1.pdf. 